\title{
Un acercamiento al estudio de la sociología y el derecho
}

\section{A closer approach to the study of sociology and law}

\author{
OROZCO-VALENCIA, Zocimo Edilberto
}

Universidad de Guadalajara, Av. Juárez \#976, Col. Centro, C.P. 44100. Guadalajara, Jalisco, México.

ID $1^{\text {er }}$ Autor: Zocimo Edilberto, Orozco-Valencia / ORC ID: 0000-0003-2503-0719, CVU CONACYT ID: 336390

DOI: $10.35429 / J L A .2019 .10 .3 .1 .6$

Recibido 01 de Octubre, 2019; Aceptado 26 de Diciembre, 2019

\section{Resumen}

Este artículo realizará un acercamiento a la sociología y al derecho. Al realizar una aproximación a los factores que determinan la observación social y su aproximación al derecho nos daremos cuenta de la cercanía de ambas ciencias. Este trabajo muestra 5 vertientes principales que son Sociología, Sociología Jurídica, Pluralismo Jurídico, Teoría Socio- Jurídica y El Concepto Sociológico del Derecho. Se visualizan distintos paradigmas que hay para una aproximación a la observación social del Derecho.

Sociología, Derecho, Estado

\begin{abstract}
This Artícle makes an approach to Sociology and Law. We could appreciate both sciences are related. 5 principal elements are commented which are Sociology, Sociology Law, Legal Pluralism, SocialLaw Theory and the social law concept. Different paradigms are showed to make a social approach of law.
\end{abstract}

Citación OROZCO-VALENCIA, Zocimo Edilberto. Un acercamiento al estudio de la sociología y el derecho. Revista de Aplicaciones del Derecho. 2019. 3-10: 1-6.

\footnotetext{
*Correspondencia al Autor (Correo Electrónico: zocimoo@hotmail.com)

$\dagger$ Investigador contribuyendo como primer autor.
} 


\section{Introducción}

En los estudios iniciales del Derecho, siempre es de resaltarse la norma jurídica, con el carácter eminentemente positivista y formalista que esto conlleva. El siguiente artículo resaltará la importancia de estudiar el aspecto sociológico del derecho, en virtud a que no se pueden desprender el estudio de la norma y la realidad social. Se expondrá el concepto sociológico del Derecho y las teorías que exponen su desarrollo

\section{Desarrollo}

El estudio del positivismo es base para explicar el concepto de derecho y el concepto del poder del Estado. DE VEGA (2012) Tomando como punto de partida... los elementos políticos, sociales, históricos y filosóficos que habían condicionado hasta entonces la exposición de la teoría del Estado, aspiró el positivísimo a la construcción de una teoría del Estado forjada única y exclusivamente desde el razonamiento jurídico. p.9.

El fundamentar el Estado por medio del poder del derecho fue un comienzo para esbozar los primeros sistemas normativos. Impero la necesidad fundamentar al Estado desde la concepción clásica del Derecho. Un Estado con poder para imponer el Derecho por medio de la fuerza.

DE VEGA (2012) Aunque Jellinek admitiera la existencia de una teoría sociológica del Estado siguiendo las premisas establecidas por Laband... su objetivo no era otro que el de establecer una teoría jurídica del Estado.pp.10-11.

Aunque Jellinek acepta el elemento sociológico del Estado, no hace sino fortalecer el concepto jurídico del Estado, dejando a un lado poder normativo de lo fáctico. DE VEGA (2012) Consecuente con esas premisas, su razonamiento se asentaría en tres postulados fundamentales. En primer lugar, en el de la positividad del derecho, que se traducía en el no reconocimiento de otro tipo de normas ajenas a las del derecho positivo. En segundo término, en la asignación al Estado del monopolio de la legislación, lo que equivalía a convertirlo en la única instancia capaz de declarar lo que es derecho... Y por último la consagración del dogma de la personalidad jurídica del Estado. p.11.
Para justificar la vida del derecho positivo era imprescindible restarle validez a cualquier otra fuente de derecho que no fuera el reconocido por el Estado.

El Estado era el único ente con capacidad para declarar el Derecho y con ello el monopolio de la aplicación del Derecho.

DE VEGA (2012) La gran construcción teórica del formalismo jurídico en la que el positivismo adquiere su máximo esplendor es obra... del vienés Kelsen. p.13. Es con Hans Kelsen y su Teoría pura del Derecho que el positivismo jurídico llega a su cúspide en el siglo $\mathrm{XX}$.

\section{El concepto Sociológico de Autoridad y Conciencia Colectiva}

Para hablar del aspecto sociológico del Derecho es importante ver el trabajo de Durkheim tal como lo hizo Habermas en su Teoría de la Acción Comunicativa.

\section{La Autoridad Moral}

Nos encontramos con que el concepto de Autoridad Moral en Durkheim se apega $a$ lo sagrado, sin embargo, no es éste mismo ajeno a relacionar a la sociedad como fuente de Autoridad. (VÁZQUEZ 2008) Para la vida social, nos vemos obligados a observar una conducta normada, restringiendo nuestras inclinaciones individuales. Este consentimiento... Responde a causas objetivas derivadas de nuestro medio social... Estos poseen una dimensión simbólica y solo pueden producirse en la medida en que la sociedad se constituye frente al individuo como una autoridad moral. p.131. En la medida que los individuos someten su comportamiento individual, mediante el consentimiento, a una conciencia colectiva dotada de simbolos, se habla de una autoridad que en este caso es la misma sociedad.

\section{La conciencia colectiva}

(VÁZQUEZ 2008) La conciencia colectiva cubre completamente la conciencia individual, vinculando a los sujetos estrechamente a su medio doméstico y sus tradiciones, delimitando el horizonte social de sus expectativas y controlando de ese modo la mayor parte de sus acciones dentro del grupo.

OROZCO-VALENCIA, Zocimo Edilberto. Un acercamiento al estudio de la sociología y el derecho. Revista de Aplicaciones del Derecho. 2019 
La conciencia colectiva muchas veces cumple la función de delimitar las conductas en el individuo. (VÁZQUEZ 2008) Si bien a Juicio de Durkheim la conciencia colectiva presenta un carácter difuso a lo largo de toda la sociedad, posee sin embargo un considerable grado de consolidación, así como rasgos característicos que le brindan estabilidad a lo largo del tiempo. p.145. La conciencia colectiva se mantiene a través de las generaciones, es algo que permanece en la sociedad aunque cambie a lo largo del tiempo.

(VÁZQUEZ 2008) La posibilidad de "conciencia" y construcción de valores trascendentes que ofrece lo social, proviene de un patrimonio alcanzado merced al fenómeno de la asociación y acumulación cultural que cada sociedad produce, generación tras generación. p.250

La conciencia colectiva es patrimonio de una sociedad así como de las generaciones presentes y futuras.

Gurivitch (VEGA ÁLVAREZ 2014) propone prescindir la lógica Individual que proviene del Derecho Romano para formular una construcción teórica que introduzca el termino de la totalidad o de lo "universal concreto" en la esfera jurídica. Esto ultimo lo lleva a formular su concepto de derecho social.p. 4

Los términos individual por colectivo o incluso universal en piensan a tomar fuerza.

Sigue Gurivitch con la idea que las personas encarnan valores y participan en la actividad de la globalidad, la idea de una colectividad pero de una que transmita valores transpersonales y que tenga una misión que cumplir.

VEGA ALVAREZ El sujeto del Derecho Social Organizado es la persona colectiva compleja, como algo distinto de las personas jurídicas unitarias y simples que absorben al individuo y lo disuelven en la colectividad. Las personas jurídicas complejas combinan unidad y multiplicad, pues sus miembros conservan su personalidad parcial en el seno de la personalidad total...p.7

\section{La sociología Jurídica}

Expone Correas (CORREAS 1993) que la sociología Jurídica es una disciplina científica que intenta explicar las causas y efectos de las normas jurídicas, para lo cual se vale de estudiar los fenómenos que son las conductas empíricamente observables conjuntamente con el efecto ideológico.

Aceptar que las relaciones sociales pueden ser causas de normas es decir que tienen fuerza suficiente para hacer ese efecto y constituirse en la explicación de estas. p.30.

(COTTERRELL 2010) Comparar distintos tipos de mecanismos informales de procesamiento de las disputas sin analizar como centralmente significativas las condiciones ideológicas bajo las cuales el procesamiento de las disputas jurídicas tienen lugar, pueden llevar a expectativas no realistas de los efectos de los procesos informales. p.62

Los tribunales y las autoridades que aplican el derecho dependen de las bases ideológicas y de los efectos de la doctrina, negar esto puede conllevar a expectativas no realistas en la aplicación del derecho.

Krawietz (1998) hace crítica a la sociología jurídica germano-occidental por su diligencia en la creación de conceptos sin prestar atención a la base teórico jurídica y jurídico sociológica.p.256

(GÓMEZ CHIQUIZA 2018) Se debe analizar el contexto de la eficacia de la norma pues sin comunicación entre las diversas disciplinas no es posible analizar y establecer si en un cuerpo normativo se presentan fenómenos como el de la eficacia simbólica en sentido restringido y el de la ineficacia material de las normas, lo que ... nos muestra que los sistemas sociales no están siendo efectivos.p. 129

Si no se analiza la norma desde un punto de vista interdisciplinar no es posible observar si la vigencia de la norma traspasa a los sistemas sociales. 


\section{EI Pluralismo Jurídico}

(IANNELLO 2015). El Pluralismo jurídico ha sido entendido como uno de los conceptos clave en la visión postmoderna del derecho, en la cual se da la coexistencia de espacios legales superpuestos interconectados e interrelacionados, y la vida de la genta está alcanzada por la inter-legalidad de dichos sistemas normativos.p.767

En el Pluralismo jurídico se acepta que varios órdenes puedan coexistir, por ejemplo, en Durkheim se entiende que las normas dependen de la estructura social.

No se entiende exclusivamente que exista una pluralidad de órdenes normativos, sino que el derecho está en la actividad cotidiana de la sociedad.

(COTTERREL 2010) En la Obra de Georges Gurvitch...La concepción del pluralismo jurídico tiende a acentuar la significativa importancia social de las ideas jurídicas como respuesta a problemas fundamentales de la interacción social bastante independientes del estado (sic) aunque desarrollados en algún nivel particular de la vida política del estado. p. 56

La idea de la importancia social en las ideas jurídicas respecto a problemas fundamentales de la interacción social es independiente del Estado en el Pluralismo Jurídico.

\section{Teoría Socio- Jurídica del Derecho}

(CALVO GARCIA 2012) La Teoría General del Derecho que defiende Tamanaha se enfoca en las relaciones entre derecho y sociedad. Consecuentemente se plantea como una aproximación transdisciplinar que nutre su perspectiva, general y compleja, con herramientas tomadas de diversos campos, entre los que se incluye la teoría social, la teoría política, la sociología, la antropología y la teoría jurídica.p. 48

La teoría socio-jurídica no se opone a un estudio interdisciplinario de la materia, así como a utilizar herramientas que son características de las ciencias particulares.
CALVO GARCIA 2010 Otro autor que ha planteado recientemente la posibilidad de un diálogo interdisciplinario entre el análisis socio-jurídico y la Teoría analítica del derecho es Denis Galligan.

La respuesta de Galligan a la pregunta sobre si la Teoría del derecho puede tener utilidad para las investigaciones soci-jurídicas recibe un rotundo sí: los conceptos de la teoría jurídica son una buena base para clasificar y dar sentido a los datos sociales.p. 386

Calvo García explicó que la utilidad de la Teoría del Derecho es limitada ya que no puede mostrarnos qué ocurre en la práctica.

\section{El Concepto Sociológico del Derecho}

(WRAVIETZ 1998) Así pues tampoco aquí (Weber) el elemento estructural decisivo de la regla jurídica es la amenaza de la sanción o la coacción, sino la expectativa normativa directamente expresada en la prescripción jurídica y con respecto a la cual se puede suponer que para el jugador, es decir para el destinatario de la norma jurídica es obligatoria, o sea que debe cumplirla. p.263.

No es la amenaza de una sanción lo que debe motivar a las personas a cumplir una norma, sino que el destinatario debe acatarla por convicción. (WRAVIETZ 1998) Luhmann rechaza expresamente ... definir al derecho a través de la violencia física en caso de aplicaciones de la norma, sino un medio para facilitar expectativas con la ayuda de las reglas del derecho. Es establecido institucionalmente con carácter de permanencia y funciona como una estructura de los sistemas sociales. p.265.

Es precisamente en la creación de una expectativa de vida donde el derecho es cumplido con voluntad de un futuro mejor; nace para perdurar, para que las futuras generaciones puedan disfrutar del trabajo de la generación actual y con ello, su carácter de permanencia. 
(KRAWIETZ 1998) Un concepto sociológico del Derecho requiere su ampliación a través de la concepción de que todo Derecho no es primordialmente un producto de la decisión de un equipo judicial, sino, sobre todo, un orden vivido, es decir, realmente vigente, normativamente eficaz, de la experiencia vital y del comportamiento de las personas, cuyas estructuras de expectativas institucionalmente establecidas con un carácter de permanencia y su distribución de derechos y deberes dependen y seguirán dependiendo de la formación social de sistemas. p.271.

Nos alejamos del concepto del derecho creado exclusivamente por los operadores jurídicos, un orden normativo vigente y eficaz se basa en la experiencia de vida y el comportamiento de las personas con lo cual se crea un sistema social.

(DELGADO ECHEVERRÍA 2018) Costa entiende que el Derecho verdaderamente vigente se encuentra en la costumbre; que todo Derecho vigente es de alguna manera costumbre. Esta Constituye el Derecho verdadero, real, vivido por la comunidad, en contraposición al derecho oficial promulgado en la Gaceta.p.76

Es importante distinguir al derecho que es producto de un medio publicado en una fuente oficial de comunicación, como lo es en México el Diario Oficial de la Federación. Es diferente de las normas que son respetadas por todos al hacerlas partes de sus actividades cotidianas o costumbre.

\section{Conclusiones}

El positivismo jurídico ha sido rebasado por nuevas concepciones teóricas de buscar un equilibrio entre el hacer una norma dotada de sanción por parte del Estado y su adecuación a la realidad social.

Si bien hay conceptos que pertenecen al campo de la sociología pura, como autoridad moral y conciencia colectiva, no por esto quiere decir que su aplicación al Derecho es nula.

La conciencia colectiva como creadora de normas es de destacarse en cuanto crea valores, tradiciones y trasciende a través de las generaciones.
Dentro del campo del estudio de la sociología y el derecho existen diferentes formas de conciliar ambas ciencias, el camino queda a discreción del investigador y de los factores sociales que intervienen en la creación de la norma jurídica.

Aunque en el pluralismo jurídico se resalta la coexistencia de distintos órdenes no por eso se deja de buscar una prevalencia para buscar el bienestar general y con esto los valores que se deben cuidar.

Se habla de que lo universal prima sobre lo individual, pero también de que lo colectivo y las personas pueden funcionar con un ente dotado de conciencia para el trabajo de la comunidad inclusive de las personas futuras generaciones.

Queda como pendiente el fortalecimiento de la Teoría Socio-Jurídica como aportadora de conceptos que la Sociología-Jurídica ha tenido a bien suplir sin ser su función principal.

Cuando se dota al individuo de una expectativa de Derecho en conjunto con una expectativa de vida es posible que su aceptación del contenido de las normas sea mayor al prevalecer el cuidado del bien común.

\section{Referencias}

Aragón Manuel, Balaguer Callejon Francisco, De Cabo Carlos, Carbonell Miguel, Carpizo Jorge, Comanducci Paolo, Ferrajoli Luigi, Ferreres Víctor, Guastini Ricardo, Gurutz Jauregui, Prieto Sanchis Luis, Rubio Llorente Francisco, Valádes Diego, De Vega Pedro, Zagrebelsky Gustavo, (2012). Teoría Constitucional, Ensayos Escogidos, México: Porrúa.

Barbeis Mauro, Barron Anne, Bengoetxsea José Ramón, Bonrino Ramírez Pablo, Botero Bernal Andrés, Bullard Alfredo, Calvo González José, Carrillo de la Rosa Yezid, Casanova Pompeau, Fabra Zamora, Goibourg, Ricardo, Jorge, Moreso José Juan, Nuñez Pozzolo Susanna, Iannello,Leiter Brian, Pablo, Rodríguez Blanco Verónica, Vaquero, Ochoa Sánchez Andrés, Orrego Sánchez Cristobal, Roca i Escoda Marta, Ricoy Rosa, Trujillo Isabel, (2015), Enciclopedia de Filosofía y Teoría del Derecho, México, Instituto de Investigaciones Jurídicas UNAM.

OROZCO-VALENCIA, Zocimo Edilberto. Un acercamiento al estudio de la sociología y el derecho. Revista de Aplicaciones del Derecho. 2019 
Calvo García, Manuel, (2012), Transformaciones jurídicas y Teoría del Derecho, Anuario de filosofía del derecho, N.28 Documento recuperado de: https://dialnet.unirioja.es/servlet/articulo?codig $\mathrm{o}=3985277$

Calvo García Manuel, (2010), ¿Cabe el Enforque Socio-Jurídico en la Teoría del Derecho? Anales de la Cátedra Francisco Suárez, N.44 Documento Recuperado de:https://dialnet.unirioja.es/servlet/articulo?co $\operatorname{digo}=4214348$

Delgado Echeverría Jesús (2018) Joaquín Costa Jurísta y Sociólogo, Universidad Carlos III de Madrid, España. Documento recuperado de: https://e-

archivo.uc3m.es/bitstream/handle/10016/26335 /joaquin_delgado_hd63_2018.pdf?sequence=1 \&isAllowed $=\mathrm{y}$

Cotterrell, R. (2010). El concepto sociológico de derecho. Revista De La Facultad De Derecho, 1(1).Documento Recuperado de https://revistas.unc.edu.ar/index.php/refade/ article/view/5877

Correas Oscar. (1993) La Sociología Jurídica, un ensayo de definición. Crítica Jurídica. Revista Latinoamericana de Política, Filosofía y Derecho. No. 12, Documento recuperado de: https://revistas-

colaboracion.juridicas.unam.mx/index.php/criti ca-juridica/article/view/3076/2876

Gómez Chiquiza Maria Eugenia (2018), El resarcimiento de los perjuicios causados en las relaciones de familia: Una breve desde la sociología y el Derecho. Revista Nuevos Paradigmas de las Ciencias Sociales Latinoamericanas. ISSN: 2346-0377. Documento Recuperado de: http://www.ilae.edu.co/ILAE_OjsRev/index.ph p/NpVol-VII-Nro14/article/download/277/429

Krawietz, Werner, (1998). El Concepto Sociológico del Derecho, Universidad de Alicante, Documento Recuperado de: http://rua.ua.es/dspace/handle/10045/10881
Vega Alvarez, Jose María Alonso, (2014), El Concepto de Derecho Social en Georges Gurvitch. Una reflexión Crítica de la Realidad y del Derecho que nace de ella. Real Academia Asturiana de Jurisprudencia, Documento Recuperado de http://www.academiaasturianadejurisprudencia. com/documentos.php

Vázquez Gutiérrez Juan Pablo, (2008), Autoridad Moral y Autonomía, México. Universidad Iberoamericana. 